\title{
Ground reaction force and spatiotemporal measurements of the gait of the mouse
}

\author{
K. A. CLARKE, L. SMART, and J. STILL \\ University of Sheffield, Sheffield, England
}

\begin{abstract}
Measurement of ground reaction forces with force plates permits quantification of the subtle changes in movement associated with a variety of pathophysiological states. The apparatus used here permits simultaneous recording of other spatial and temporal characteristics of gait. Since the mouse is becoming increasingly important for modeling human disorders, we have recorded ground reaction forces $(P)$ in this species. To date, we have measured the vertical component $\left(P_{z}\right)$ and found it to be similar to that of other species, in that a greater $P_{z}$ is exerted via the forelimb, although the areas under the force/time curves, are not different. Different points of discontinuity are seen in the forelimb and hindlimb curves, indicating differential deployment. $P_{z}$ varies consistently with stance time and is consistent across the adult life of the mouse. It is suggested that the technique has potential for the study of a variety of mouse models of disease and of their therapies.
\end{abstract}

The use of force plates for the measurement of ground reaction forces during locomotion provides a sensitive and noninvasive method for analyzing the relative contribution of each limb to the different phases of the gait cycle (Biewener, 1983; Biewener, Alexander, \& Heglund, 1981; Muir \& Whishaw, 1999a, 1999b, 2000). However, this approach does not permit simultaneous viewing of the animal from below. Therefore, one cannot determine the nature of the individual paw contacts and, thus, cannot with accuracy match the forces produced to the spatiotemporal aspects of the contact. To this end, we previously adapted the internally illuminated glass plate technique for producing a pedobarograph (Betts \& Duckworth, 1978) and mounted it on a load cell, for analysis of the gait of the rat. This allows subtle changes in gait to be assessed and applied to the study of pathophysiologicalstates and potential therapies (Clarke, Heitmeyer, Smith, \& Taiwo, 1997; Muir \& Whishaw, 1999a, 1999b). The smaller the animal, however, the more technically difficult the measurement becomes. The current emphasis on mouse models of human diseases has motivated us more recently (Clarke \& Still, 1999) to adapt the technique to examination of the gait of the mouse in order to give us a platform for future measurements in such models.

\section{METHOD}

Observations were made of the spontaneous locomotion of 10 adult Swiss (MF1 subtype) mice ( 5 male, 5 female) drawn from two

This work was performed with the generous support of a grant from the Animal Procedures Committee of the Home Office, U.K., under Project Licence PPL 50/1177. Correspondence concerning this article should be addressed to K. A. Clarke, Department of Biomedical Science, University of Sheffield, Western Bank, Sheffield S10 2TN, England (e-mail: k.a.clarke@ sheffield.ac.uk). litters (mean age at start of study, 30 weeks). They were kept in a 12:12-h light:dark cycle and were allowed food and water ad lib. Each mouse was weighed (mean weight at start of study, $45 \mathrm{~g}$ ), and its gait during spontaneous locomotion was recorded on three separate occasions over an 8-week period (or for the longitudinal part of the study, 13 times over a 12-month period). Each animal was introduced separately to the apparatus and was allowed to move freely for 3-5 min., with all observations being made between 10.00 and $13.00 \mathrm{~h}$ to minimize any effects of diurnal variability.

Data from the 3 (or 13) runs for each animal were averaged. The apparatus for measuring mouse reaction force was developed from that used previously in the rat. This latter has been described before (Clarke et al., 1997) and is shown in Figure 1. Briefly, it consists of a glass-floored Plexiglas tunnel, $98 \times 4 \times 6.5 \mathrm{~cm}(1 \times \mathrm{w} \times \mathrm{h})$, located in a dedicated, soundproofed laboratory, with a central $30-\mathrm{cm}$ section of the glass floor (high-quality $5-\mathrm{mm}$ glass with polished edges; Avimo Ltd., Taunton, U.K.) divided into six $10 \times 8 \mathrm{~cm}$ pieces. Four of these sections are internally illuminated using cowled 8-W fluorescent tubes applied to the outer edge of the glass, so that paw contact points can be better seen, whereas four of the sections of glass floor (two common with those internally illuminated) form load cell platforms (Uniweigh FSO cell, range 0-500 g; Maywood Instruments Ltd., U.K.) to determine $P_{Z}$. This type of cell was chosen because it is immune to eccentric loading of its platform. The load cell output was captured by a data acquisition system sampling at $200 \mathrm{~Hz}$ (Powerlab 400 series) and was analyzed in an Acer PC running Chart for Windows 95 software (AD Instruments Ltd., Australia).

One problem that can arise with the use of force plates to examine the gait of small rodents is the production of vibrational noise as each limb impacts the surface. Various techniques are used to reduce this (Handley, Ross, \& Carr, 1998). Such interference, however, is not a problem in our load-cell-based system. Here, the force can also be calculated by the alternative method of calibrating the pixel brightness of the paw contact points in terms of pressures, which are not susceptible to vibrational interference (Betts \& Duckworth, 1978; Clarke, 1995). The force profiles produced by the two methods are the same, with a correlation coefficient of .92 (Clarke, 1995). Furthermore, vibration induced by tapping the index finger on the platform at a velocity twice that of mouse limb placing is not seen until the force applied reaches 7 times the peak typically achieved by the walking mouse. At this point, a $38-\mathrm{Hz}$ vibrational noise 


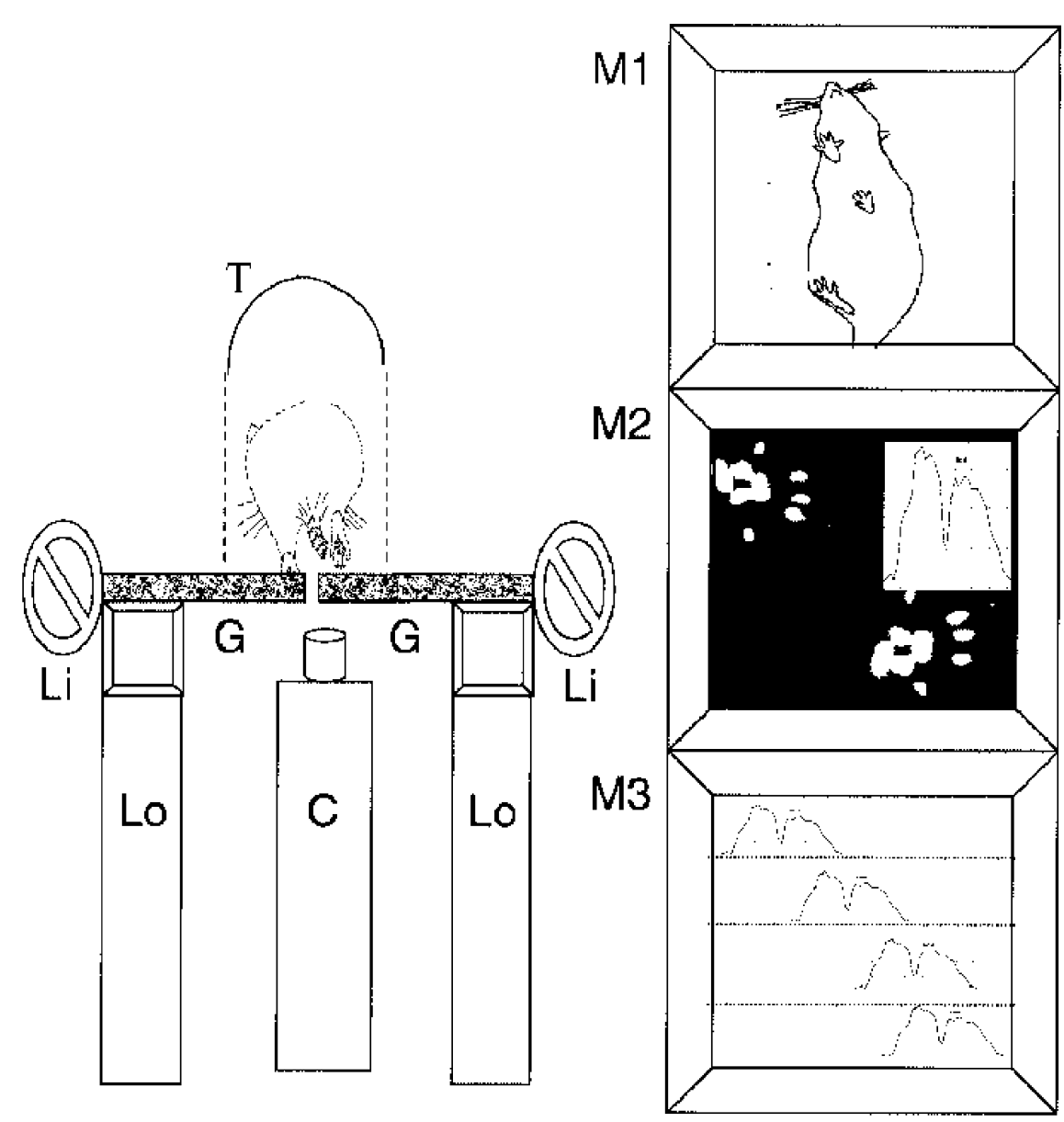

Figure 1. The apparatus used to collect spatiotemporal and vertical reaction force data consists of one Plexiglas tunnel (T) suspended over a glass walkway (G), internally illuminated by lights (Li) placed along the edge, under which are placed two video cameras $(\mathrm{C})$. Lo, load cell. Their output goes via a video splitter to monitors M1 (general view of walking mouse) and M2 (detailed view of paw contacts). M2 also shows force profiles associated with those contacts. M3 is the monitor of the data acquisition system showing the progress of the mouse across the four load cells. Note that the tunnel and mouse are shown at larger scale than the rest of diagram for clarity.

is produced, but with an amplitude of only $3 \%$ of the peak signal noise.

To restrict data collection to the times during which the mouse was moving across the load cell platforms, a trigger threshold switch (Sheffield BMS Electronics) was incorporated into the data input line that would transmit only when any one of the load cells had been triggered by a weight greater than $5 \mathrm{~g}$. A TTL pulse was generated that activated a 500-msec pretrigger recording period by the Powerlab, to ensure full data collection. Data were saved to an Iomega zip drive for subsequent analysis. A camera positioned below the central part of the chamber recorded underviews of the walking mouse. The images of the mouse were recorded on tape (Fuji 180) on a Panasonic AG 7350 video cassette recorder. Output from the data acquisition system, including $P_{Z}$ and a 10-msec timing pulse, were passed through a VGA to a video converter (Hama Trilock plus, Bayern, Germany) and were superimposed as a window in the video of the mouse walking, using a Multiview 2 Video Effector (VIS, U.K.). Thus, particular load profiles could be assigned to the limbs transmitting them and correlated with the load data stored in the Chart files, on which detailed analysis could then be performed. Recordings were analyzed for the temporal aspects of gait on a field-by-field basis (i.e., every $20 \mathrm{msec}$ ), allowing an average accuracy to $\pm 10 \mathrm{msec}$. That this degree of temporal resolution was adequate was confirmed from a sample of simultaneous and independent measurements taken from the time axis of the Chart recordings of the vertical reaction forces $\left(P_{Z}\right)$, which was sampled at $200 \mathrm{~Hz}$ and from which stance times could be independently measured. These timings yielded a correlation coefficient of .98 .

Mean values for each variable for individual mice over the 3 (or 13) sessions in the apparatus were calculated. Values were discarded from sessions producing fewer than three values for an individual limb. From these means, a group mean and an SEM were calculated for the whole sample of mice. Differences between variables calculated for fore- and hindlimbs were analyzed using Wilcoxon signed ranks tests for related samples.

\section{RESULTS}

The peak vertical reaction forces transmitted via the left and right sides of the mouse are symmetrical, but 


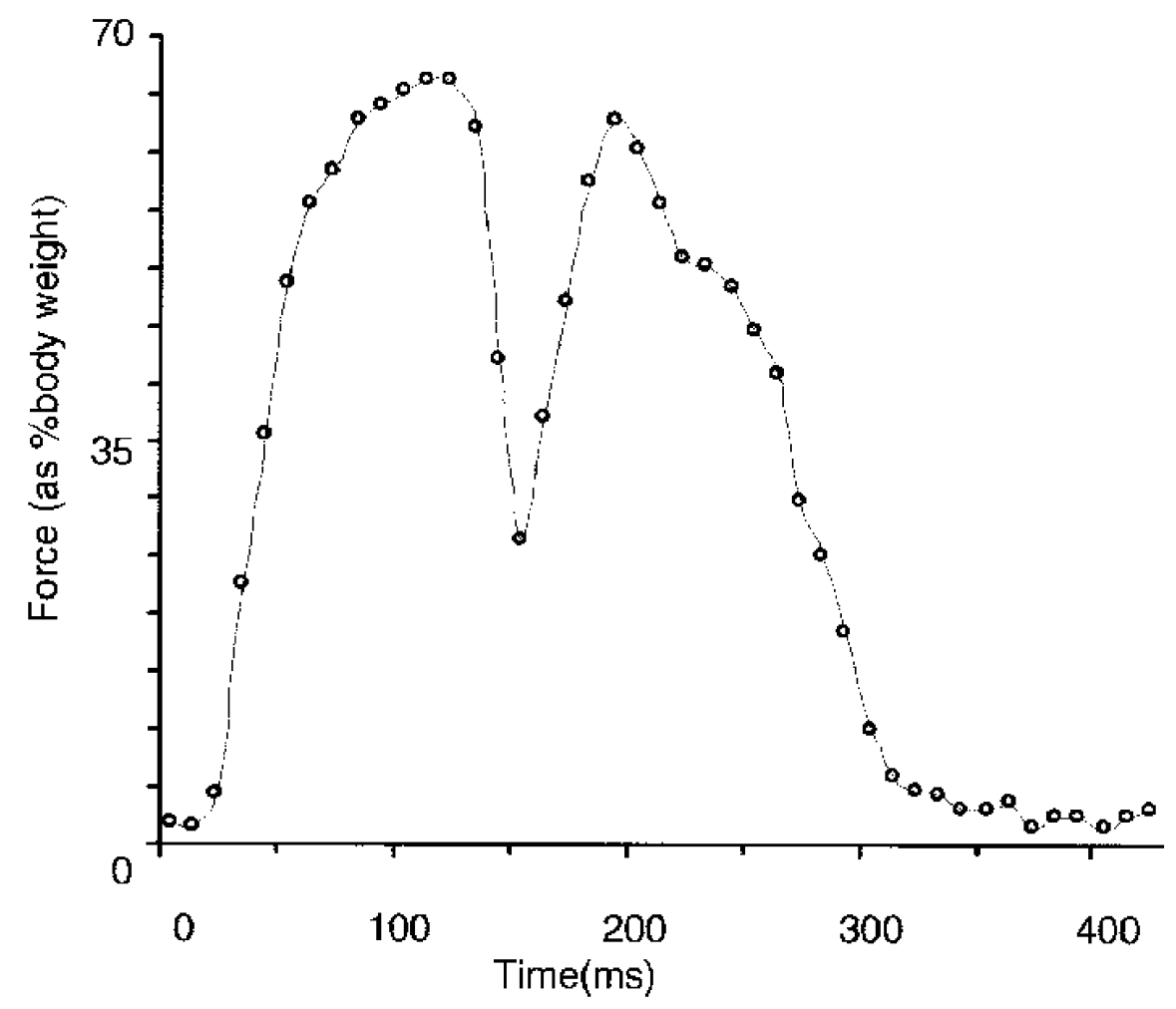

Figure 2. Variation of the vertical reaction force $\left(P_{Z}\right)$, with time for typical forelimb (left-hand peak) and hindlimb (right-hand peak) placements on a load cell.

those via the forelimbs are about $5 \%$ greater than those via the hindlimbs $(63.5 \% \pm 0.6 \%$ and $58.4 \% \pm 0.7 \%$, mean $\pm 1 S E M$, of body weight, respectively; $Z=4.9, p<$ $.0001)$, whereas the areas under the vertical load curves were not significantly different at $7.2 \pm 0.2$ and $7.1 \pm$ 0.2 (gram seconds normalized to percentage of body weight), since the latter has a longer stance time (164 \pm 6.1 and $190 \pm 7.7 \mathrm{msec}$, respectively). Typical examples are shown in Figure 2. It appears that discontinuities are present in the gradients of these profiles. To verify this, traces for each mouse produced at mean velocity were examined and normalized to allow comparison with those from other mice. This is shown in Figure 3.

The $P_{z}$ curves for the forelimb and the hindlimb in mice traveling at or around average velocity consist of three distinct phases for each, lasting from $12.4 \%$ to $27 \%$ of the total combined stance time. The major difference is that for forelimb, two of the phases occur prior to the peak, whereas for the hindlimb, two occur after the peak.

An inverse variation in $P_{z}$ with stance time was noted (Figure 4). The values for the forelimb and the hindlimb for a stance as short as $130 \mathrm{msec}$ were $69.4 \%( \pm 1.1 \%)$ and $65.8 \%( \pm 1.5 \%)$ whereas at $270 \mathrm{msec}$, they were $59.3 \%( \pm 1.3 \%)$ and $53.4 \%( \pm 0.9 \%)$. No significant differences were found between males and females for any of these variables. It appears that the differential between forelimb and hindlimb $P_{z}$ becomes less with decreasing stance time.
The consistency of $P_{z}$ was assessed by measuring it at intervals over a 12-month period of the adult life of the mouse. No significant changes occurred. $P_{z}$ via forelimb remained at $64.6 \% \pm 2 \%$, that via hindlimb at $60.7 \% \pm$ $1.9 \%$, and the difference between the two at $4.5 \% \pm$ $0.9 \%$ (mean $\pm S D, n=13$ ) of body weight.

\section{DISCUSSION}

We have shown that it is technically possible to measure vertical reaction forces produced during mouse gait and to associate them with various spatiotemporal aspects. Overall, those forces via the forelimb are around $5 \%$ greater than those via the hindlimb. This makes the gait of the mouse different from that of the rat, for which the greater force is via the hindlimb (Clarke et al., 1997). It is consistent throughout a considerable portion of adult life, making it suitable for the study of long-term neural and other pathologies. Normalization of the force profiles and identification of the turning points in their gradients when the mouse is traveling at average velocity indicates that each of the profiles consists of three phases. The major difference between the fore- and the hindlimb is that, in the former, two of these phases occur prior to the peak force, which therefore occurs relatively late during the stance, whereas in the hindlimb, only one occurs before the peak, which therefore occurs relatively early in the stance. However, some caution should be ex- 


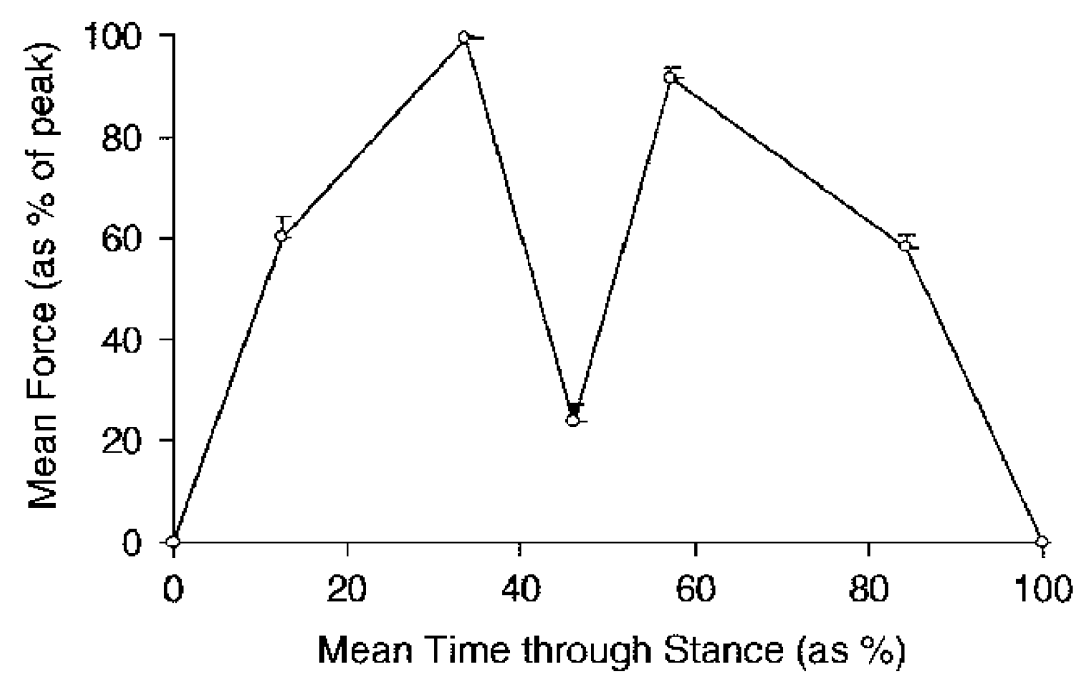

Figure 3. Variation of vertical reaction force $\left(P_{Z}\right)$, with time for forelimb (left-hand peak) and hindlimb (right-hand peak) placements on the load cells. Turning points in the gradients were identified by eye, and to allow combination of data, values obtained were normalized to percentages of forelimb peak and percentages of time through stance. Each point is a mean $( \pm 1 S E M, n=10)$.

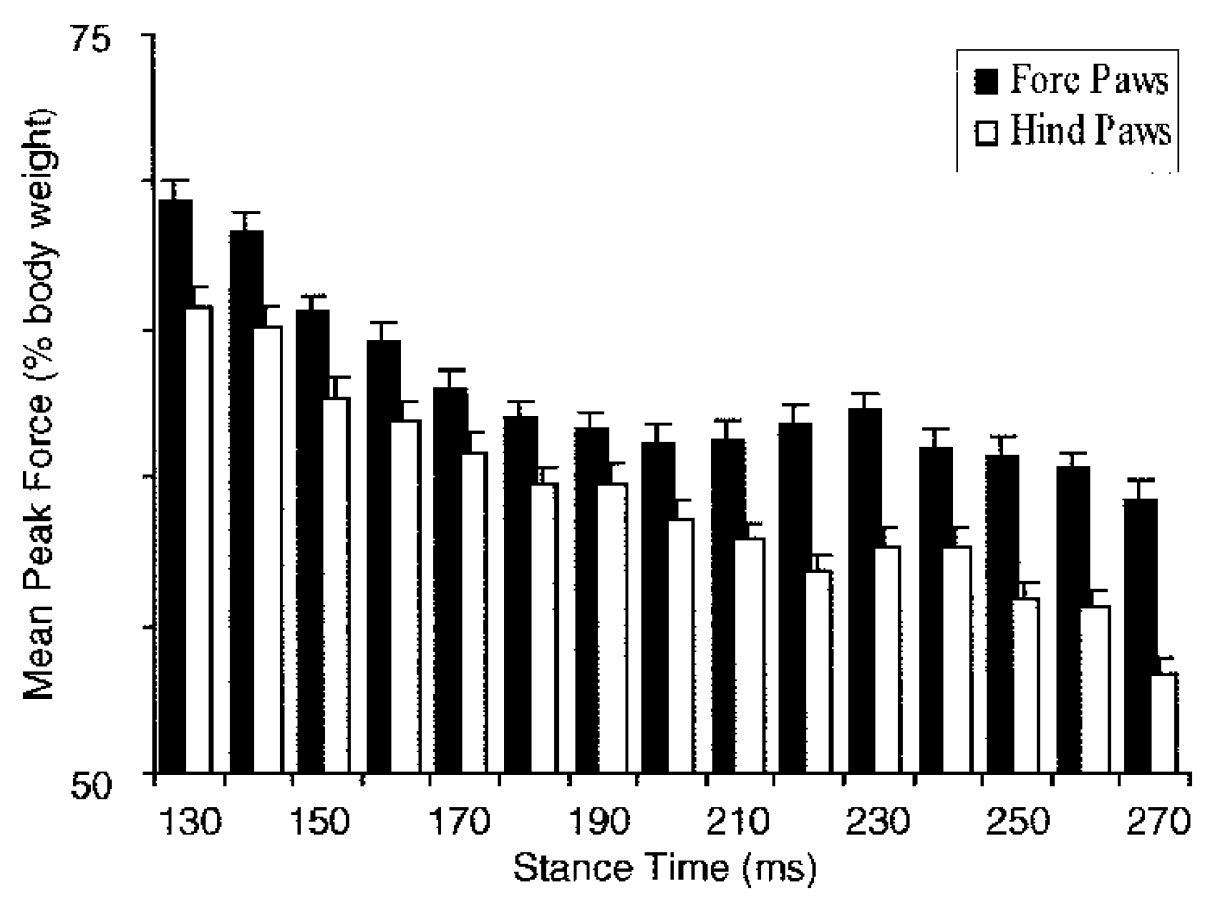

Figure 4. Variation of vertical reaction force (mean $+1 S E M, n=10$ ), with stance time for forelimbs (filled columns) and hindlimbs (open columns).

ercised in this interpretation. For most of its gait patterns, the mouse, like many other quadrupeds, deploys successive ipsilateral forelimb/hindlimb paw placements within a small area of floor, resulting in the same load cell platform's being activated by both. Thus, the end of the forelimb stance and the start of the hindlimb stance overlap, obscuring what may be happening during this 20 msec or so. There may be transitions hidden in this short period that we do not see. However, from the limited data we have seen when forepaw and hindpaw do fall on separate load cells, this does not appear to be a problem. Also, assessment of the force by the alternative method of analyzing the pixel brightness of the contact points (Clarke, 1995) would suggest that this is not a problem, 
at least in the rat. We are currently identifying the paw contact features associated with the different phases. In the rat, measurement of reaction forces has proved useful for following a variety of pathological states. We have shown that the technique has similar potential in the mouse, and we are currently examining a number of transgenic and knockout mice from this perspective.

\section{REFERENCES}

Betts, R. P., \& Duckworth, T. (1978). A device for measuring the plantar pressures under the sole of the foot. Engineering in Medicine, 7, 223-228.

Biewener, A. (1983). Locomotory stresses in the limb bones of two small mammals-the ground-squirrel and chipmunk. Journal of Experimental Biology, 103, 131-154.

Biewener, A., Alexander, R. M., \& Heglund, N. C. (1981). Energy storage in the hopping of kangaroo rats (Dipodomys spectabilis). Journal of Zoology, 195, 369-383.

Clarke, K. A. (1995). Differential fore- and hindpaw force transmission in the walking rat. Physiology \& Behavior, 58, 415-419.
Clarke, K. A., Heitmeyer, S. A., Smith, A. G., \& Taiwo, Y. O. (1997). Gait analysis in a rat model of osteoarthrosis. Physiology \& Behavior, 62, 951-954.

Clarke, K. A., \& Still. J. (1999). Gait analysis in the mouse. Physiology \& Behavior, 66, 723-729.

HandLey, D. E., Ross, J. F., \& CARR, G. J. (1998). A force plate system for measuring low magnitude reaction forces in small laboratory animals. Physiology \& Behavior, 64, 661-669.

MuIR, G. D., \& WHISHAW, I. Q. (1999a). Complete locomotor recovery following corticospinal tract lesions: Measurement of ground reaction forces during overground locomotion in rats. Behavioural Brain Research, 103, 45-53.

Muir, G. D., \& Whishaw, I. Q. (1999b). Ground reaction forces in locomoting hemi-Parkinsonian rats: A definitive test for impairments and compensations. Experimental Brain Research, 126, 307-314.

Muir, G. D., \& Whishaw, I. Q. (2000). Red nucleus lesions impair overground locomotion in rats: A kinetic analysis. European Journal of Neuroscience, 12, 1113-1122.

(Manuscript received September 25, 2000; revision accepted for publication May 16, 2001.) 